\title{
Comparison of outcomes following a cytological or histological diagnosis of malignant mesothelioma
}

Sanjeevan Muruganandan ${ }^{1,2,3}$, Helman Alfonso ${ }^{4}$, Peter Franklin ${ }^{5}$, Keith Shilkin ${ }^{6}$, Amanda Segal ${ }^{6}$, Nola Olsen ${ }^{5}$, Alison Reid ${ }^{4}$, Nick de Klerk ${ }^{5,7}$, AW (Bill) Musk ${ }^{1,2,5}$ and Fraser Brims ${ }^{\star 1,1,3,8}$

${ }^{1}$ Department of Respiratory Medicine, Sir Charles Gairdner Hospital, Perth, WA, Australia; ${ }^{2}$ School of Medicine and Pharmacology, University of Western Australia, Perth, WA, Australia; ${ }^{3}$ Institute for Respiratory Health, Perth, WA, Australia; ${ }^{4}$ School of Public Health, Faculty of Health Sciences, Curtin University, Perth, WA, Australia; ${ }^{5}$ School of Population Health, University of Western Australia, Perth, WA, Australia; ${ }^{6}$ PathWest, Queen Elizabeth II Medical Centre, Perth, WA, Australia; ${ }^{7}$ Telethon Kids Institute, University of Western Australia, Perth, WA, Australia and ${ }^{8}$ Curtin Medical School, Faculty of Health Sciences, Curtin University, Perth, WA, Australia

Background: Survival with the epithelioid subtype of malignant mesothelioma (MM) is longer than the biphasic or sarcomatoid subtypes. There is concern that cytology-diagnosed epithelioid MM may underdiagnose the biphasic subtype. This study examines survival differences between patients with epithelioid MM diagnosed by cytology only and other subtypes diagnosed by histology.

Methods: Demographics, diagnosis method, MM subtype and survival were extracted from the Western Australia (WA) Mesothelioma Registry, which records details of all MM cases occurring in WA.

Results: A total of $2024 \mathrm{MM}$ cases were identified over 42 years. One thousand seven hundred forty-four (86.2\%) were male, median (IQR) age was 68.6 (60.4-77.0) years. A total of 1212 (59.9\%) cases were identified as epithelioid subtype of which 499 (41.2\%) were diagnosed using fluid cytology only. Those with a cytology-only diagnosis were older than the histology group (median 70.2 vs 67.6 years, $P<0.001$ ), but median survival was similar (cytology 10.6 (5.5-19.2) vs histology 11.1 (4.8-19.8) months, $P=0.727$ ) and Cox regression modelling adjusting for age, sex, site and time since first exposure showed no difference in survival between the different diagnostic approaches.

Conclusions: Survival of cytologically and histologically diagnosed epithelioid MM cases does not differ. A diagnostic tap should be considered adequate to diagnose epithelioid MM without need for further invasive testing.

Malignant mesothelioma (MM) is an uncommon, aggressive cancer that affects the pleural and peritoneal tissues and is caused by the inhalation of asbestos fibres. Latency from first exposure varies from 15 to 50 years (Olsen et al, 2011), there is no cure and median survival is 10-12 months (Brims and Maskell, 2013). The incidence of $\mathrm{MM}$ varies internationally and reflects historical utilisation of asbestos in the twentieth century (WHO, 2010). Western Australia (WA) did have the highest incidence per capita of $\mathrm{MM}$ in the world principally due to the mining and widespread utilisation of crocidolite from the mining of blue asbestos at Wittenoom during the last century (Musk et al, 1992; Reid et al, 2014). Asbestos utilisation continues to increase in many developing countries (Brims, 2009; Kazan-Allen, 2005).

Malignant mesothelioma has distinctive histological subtypes: epithelioid, sarcomatoid and biphasic (composed of both epithelioid and sarcomatoid components). The diagnosis of MM using

*Correspondence: Associate Professor Dr F Brims; E-mail: fraser.brims@curtin.edu.au

Received 5 December 2016; accepted 16 January 2017; published online 14 February 2017

(C) 2017 Cancer Research UK. All rights reserved 0007-0920/17 
cytology only has been controversial. According to the Consensus Statement of the International Mesothelioma Interest Group, 'The diagnosis of MM must always be based on the results obtained from an adequate biopsy in the context of appropriate clinical, radiologic and surgical findings' (Husain et al, 2013). The British Thoracic Society guidelines offer a more pragmatic approach stating, 'a biopsy is recommended if there is doubt about the diagnosis... as cytology may be unreliable' (British Thoracic Society Standards of Care, 2007). Pooled data from different studies demonstrate a $30 \%$ sensitivity for the cytological diagnosis of MM (Renshaw et al, 1997b; Churg et al, 2000; Stahel et al, 2008), which is considered to be too low to be useful as a diagnostic tool (Husain et al, 2013). One of the reasons for this is the different biological behaviour of the pathological subtypes of MM. Epithelioid MM (the most common form, $\sim 60 \%$ of cases) more readily sheds cells into the pleural or peritoneal space and these can be identified on cytological examination. By contrast, sarcomatoid MM cells generally do not shed into the pleural or peritoneal space, leading to a poor cytological diagnostic yield for the sarcomatoid subtype and the possibility of misclassification of biphasic MM as epithelioid MM using the cytology-only approach. A cytological diagnosis of MM, however, has practical advantages including that it is minimally invasive, easily performed and inexpensive. It has been previously demonstrated that in an experienced laboratory a cytological diagnosis of MM can be accurately and reliably made (Hjerpe et al, 2015 ) with a definitive diagnosis of $\mathrm{MM}$ in $73 \%$ of all cases, with no false-positive diagnoses of malignancy (Segal et al, 2013).

The pathological subtype of MM has a strong influence on survival with the epithelioid type associated with a longer survival as compared with biphasic and sarcomatoid MM (Musk et al, 2011; Brims et al, 2016). Although chemotherapeutic approaches are limited in MM, it is generally accepted that there are better responses to treatment with the epithelioid subtype (Billé et al, 2015). There is, therefore, a clinical advantage to identify the mesothelioma pathological subtype, with prognostic and therapeutic implications. This study aimed to analyse survival between cytology-only and histologically diagnosed epithelioid MM to establish if a clinically meaningful difference exists between patients diagnosed by the two techniques.

\section{MATERIALS AND METHODS}

Case ascertainment. The Western Australia Mesothelioma Registry (WAMR) was established in 1962 and is linked to the Western Australian Cancer Registry, which records all cancer types and survival data for the state. Cancer is a notifiable disease in WA and all incident cases of MM are investigated. The WAMR research officer gathers information from pathology laboratories, hospitals and clinics, and all cases are reviewed by a committee that includes an expert pathologist, a respiratory physician, an epidemiologist and an occupational physician to confirm the diagnosis and record all available information on past asbestos exposure. All cases have clinical, radiological and pathological features discussed. In cases of ambiguity, the original sample was requested and (re)analysed in our institution's laboratory. Where there is remaining doubt in the diagnosis, the case is regarded as 'suspected' and the data were not included in this analysis. For each case, age, sex, site of disease, dates and methods of diagnosis, histological subtype, date of death and available history of asbestos exposure are sought and documented. When additional new diagnostic material was obtained, records were changed or updated to reflect the subtype or means of diagnosis. Therapeutic information and staging data are not available on the database.

Pathological diagnosis. Every pathology report was reviewed (by KBS) to confirm the diagnosis with all available cytology, histology, necropsy, immunohistochemistry and/or electron-microscopy (EM) reports considered for assessment. Diagnostic criteria did not change significantly over the study period, although the increasing use of immunohistochemical stains over the last 15 years essentially replaced the use of EM. All specimens have routine smears and a cell block prepared following cytocentrifugation, with $5 \mathrm{ml}$ of the supernatant frozen and stored. The cell block specimen ensures that paraffin-embedded material is available for immunohistochemical and molecular studies. The subtype of mesothelioma was recorded as epithelioid, sarcomatoid, biphasic or not defined. Cases diagnosed by effusion cytology are accepted as true cases and recorded as epithelioid (Segal et al, 2013).

Data analysis. Data was extracted on all confirmed MM cases available on the WAMR for which survival data and means of diagnosis was determined. For this study, cases considered to be epithelioid were subdivided by diagnostic method into 'epithelioid - cytology' and 'epithelioid - histology' to allow comparison. Overall, survival was calculated from the date of diagnosis (from pathological report) to date of recorded death on the WA Cancer Registry; the date of censoring was 31 December 2012.

Continuous variables were described by their means and standard deviations and compared using $t$-tests. Proportions were used to summarise categorical variables and compared using $\chi^{2}$-tests. Survival between groups was compared using the log rank test and Cox proportional hazards regression was used to estimate the association between the different diagnostic approaches, other explanatory variables and survival. Kaplan-Meier survival curves were also produced and analysed by decade of diagnosis. All statistical tests reported are two-tailed. Statistical calculations and data manipulation were performed using SPSS (Version 23.0. Armonk, NY: IBM Corp).

The study was approved by the Department of Health WA Human Research Ethics Committee.

\section{RESULTS}

A total of $2024(1744(86.2 \%)$ male) cases of MM were identified over a period of 42 years, median age at diagnosis was 68.6 (interquartile range (IQR): 60.4-77.0) years, 110 were alive at end of follow-up. Pleural MM was present in 1888 (93.3\%) cases. Of those with a known exposure source $(n=1750), 1467(83.8 \%)$ of cases had occupational exposure, ex-Wittenoom miners and township residents made up $25.9 \%$ of the cohort.

A total of $1212(59.9 \%)$ cases had epithelioid subtype MM of which 499 (41.2\%) were diagnosed using effusion cytology only. When comparing the diagnostic group for epithelioid MM, those with a cytological diagnosis only were older, but there was no difference in any other parameters (Table 1).

The different MM subtypes with median survival times are presented in Table 2. Survival varied statistically significant between all groups $(P<0.0001$ by log rank test: Figure 1$)$. There was no statistically significant difference in overall survival between the epithelioid - histology and cytology-only subgroups $(P=0.73$, by log rank test), but there was a statistically significant difference when comparing cytology-only to biphasic or sarcomatoid subtype survival $(P=0.0001)$. When comparing survival stratified by decade of diagnosis there was a trend towards increasing median survival, Table 3 and Figure 2.

Peritoneal MM was present in 136 (6.7\%) cases. The median survival in this group was less than pleural MM (4.5 (IQR 2.3-9.0) vs 8.8 (IQR 3.9-17.1) months, $P<0.005$ ). There were 102 cases of epithelioid peritoneal MM. Median age at diagnosis (66.4 years, IQR: 58.4-73.7) was similar to the overall cohort and did not vary between diagnostic group (histology 67.7 vs cytology-only 64.0 years, $P=0.58$ ). There was no statistically significant survival 
Table 1. Characteristics of the epithelioid MM cases according to method of diagnosis

\begin{tabular}{|c|c|c|c|}
\hline & $\begin{array}{l}\text { Epithelioid-histology (total } \\
\qquad n=713 \text { ) }\end{array}$ & $\begin{array}{l}\text { Epithelioid-cytology (total } \\
\qquad n=499)\end{array}$ & $P$-value \\
\hline Median age at diagnosis, years (IQR) & $67.6(59.3-75.5)$ & $70.2(61.5-78.6)$ & $<0.001$ \\
\hline Male & $606(85.3)$ & $412(82.6)$ & 0.204 \\
\hline Pleural & $660(92.6)$ & $450(90.2)$ & 0.141 \\
\hline Occupational exposure & $496(80.8)$ & $357(81.1)$ & 0.885 \\
\hline Wittenoom worker & $176(24.7)$ & $135(27.1)$ & 0.353 \\
\hline Time from first exposure to diagnosis, years (s.d.) & $43.3(11.8)$ & $44.7(11.3)$ & 0.079 \\
\hline
\end{tabular}

\begin{tabular}{|c|c|c|}
\hline $\begin{array}{l}\text { Mesothelioma } \\
\text { subtype }\end{array}$ & n (\%) & $\begin{array}{c}\text { Survival, months } \\
\text { (IQR) }\end{array}$ \\
\hline Epithelioid - histology & $713(35.2)$ & $10.6(5.5-19.2)$ \\
\hline Epithelioid - cytology & $499(24.7)$ & $11.1(4.8-19.8)$ \\
\hline Biphasic & 412 (20.4\%) & $7.8(3.7-15.1)$ \\
\hline Sarcomatoid & 260 (12.8\%) & $4.0(1.8-7.5)$ \\
\hline Not defined & 140 (6.9\%) & $4.4(1.2-11.7)$ \\
\hline
\end{tabular}

difference between the groups (histology 5.0 vs cytology-only 4.5 months, $P=0.43$ by $\log$ rank test).

When restricting analyses to epithelioid-only MM using a Cox proportional hazards model, increasing age, male sex and peritoneal MM were significantly associated with a shorter survival, whereas method of diagnosis was not (Table 4).

\section{DISCUSSION}

This study demonstrates that cases with a cytology-only diagnosis of epithelioid MM have a similar survival outcome when compared with those with a histological-derived diagnosis of epithelioid MM. In addition, there is a consistent and clear separation of survival curves of (cytologically or histologically diagnosed) epithelioid MM from histologically diagnosed biphasic MM, which indicates that in this population there appears to be no clinically meaningful difference between cytologically and histologically diagnosed epithelioid MM.

Current international guidelines cite historical concerns with regard to using cytology as a means of diagnosing MM and it is widely accepted that this method is not useful for the diagnosis of sarcomatoid-containing tumours. There is accumulating evidence that a cytological diagnosis of MM supported with supplementary techniques such as immunohistochemistry is as reliable as histopathological diagnosis (Rakha et al, 2010). Concern has been raised that a cytologically derived MM diagnosis may misclassify biphasic MM as epithelioid, because the sarcomatoid component cells are not shed into the pleural space, leading to errant classification. By using survival as a surrogate for different MM histology, the current study does not support this misclassification notion. In contrast, the results of this study demonstrate that cases with a diagnosis of epithelioid MM using cytology only have no significant difference in overall survival as compared with cases with histologically diagnosed epithelioid MM and, importantly, there is a statistically significant separation in survival outcome when compared with biphasic or sarcomatoid MM.

After appropriate clinical history and imaging, a diagnostic aspiration is widely accepted as the first invasive investigation for

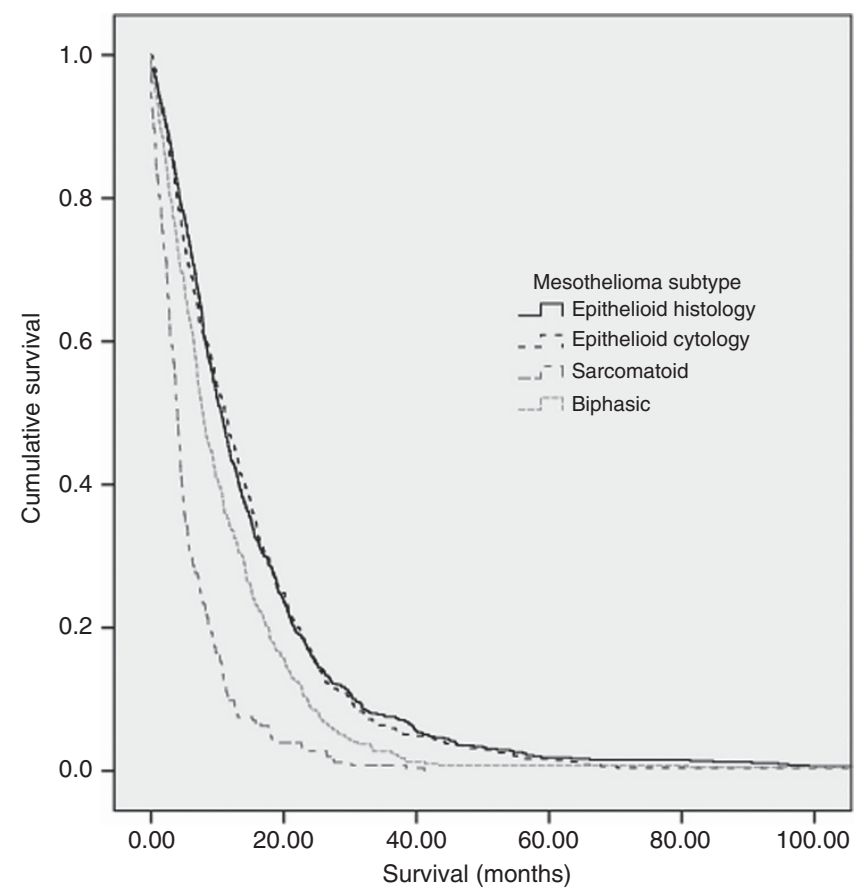

Figure 1. Kaplan-Meier survival curves for 1884 cases of MM separated by subtype. For ease of interpretation, 'not defined' cases $(n=140)$ have been omitted.

an unexplained pleural or peritoneal effusion in most cases. This allows a cytological analysis and a diagnosis of a malignant pleural/ peritoneal effusion in many cases. The importance of this current study is that with appropriate cytopathological expertise and technique, a diagnosis of epithelioid MM can be confidently made, potentially with no requirement for further invasive biopsies using an image-guided approach or thoracoscopic techniques. A previous audit of practice in our institution demonstrated that on average a cytology report was available 29 days before subsequent tissue diagnosis for MM cases (Segal et al, 2013). Therefore, cytological analysis has clear advantages for patients and healthcare resources. Clinicians can reliably inform patients of the diagnosis and prognosis with the knowledge that there is no clinically meaningful difference between overall survival with either diagnostic technique.

Effusion cytology is minimally invasive and inexpensive, which are further important considerations in this population given the limited life expectancy. A cytological diagnosis of MM should fulfil one of the following criteria: indisputable malignant cells on cytomorphological criteria, which demonstrate mesothelial phenotype confirmed by ancillary testing; or cytomorphological features which are not unequivocally malignant, but with ancillary techniques confirming malignancy and a mesothelial phenotype (Hjerpe et al, 2015). The diagnosis of mesothelioma in effusion 
Table 3. Median survival in months (IQR) of epithelioid mesothelioma cases by stratified decade of diagnosis and diagnostic approach

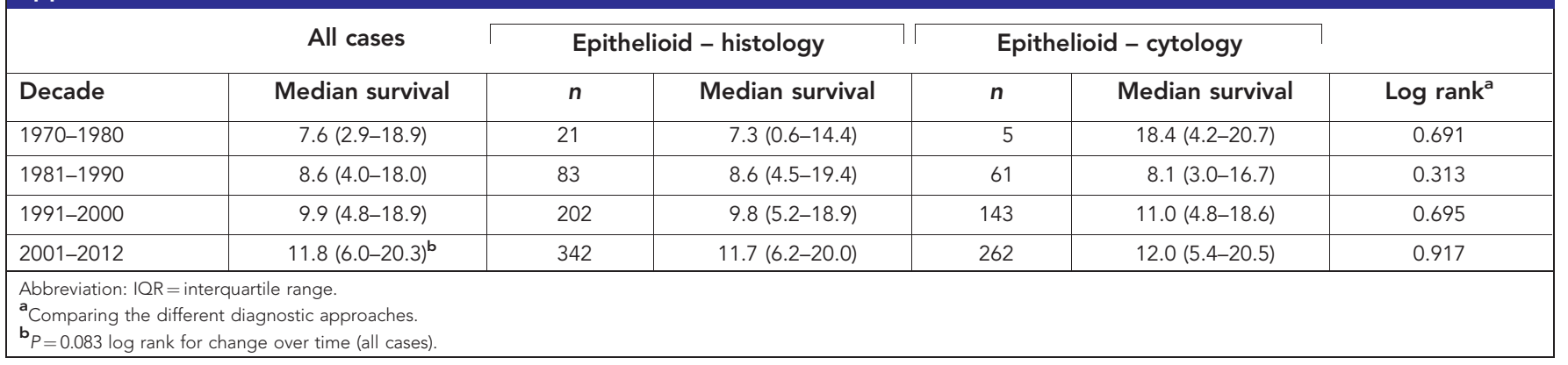

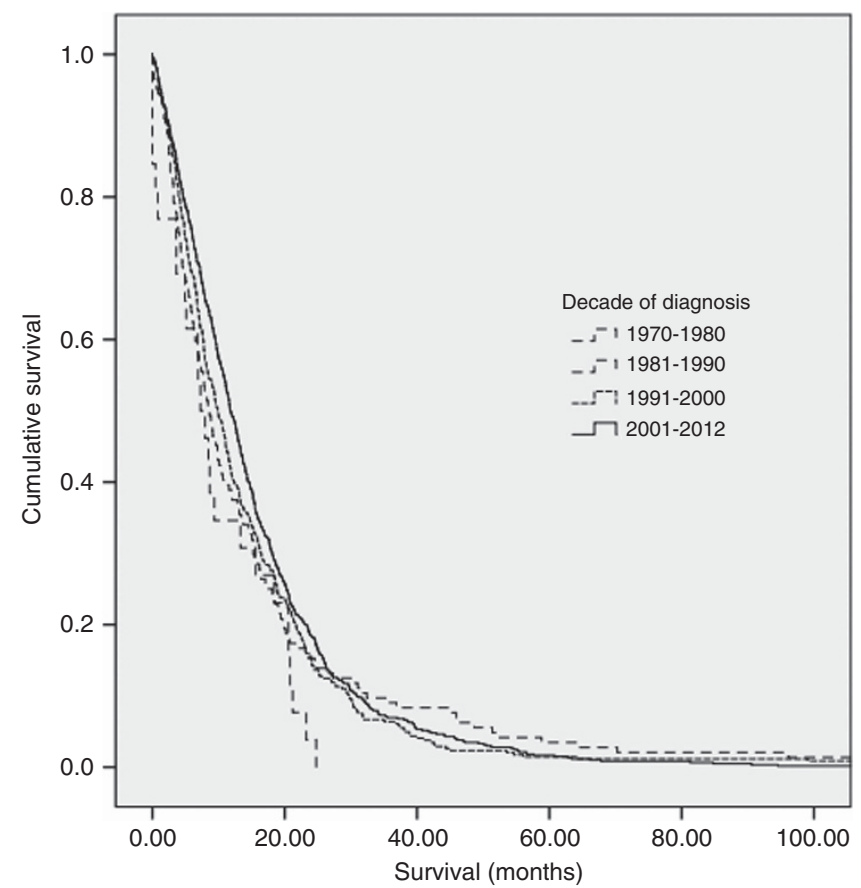

Figure 2. Kaplan-Meier survival curves for 1212 cases of epithelioid MM separated by decade of diagnosis.

samples is made using a combination of morphological features and immunohistochemistry. The main change that has occurred over the past 20 years is the current availability of reliable immunohistochemical mesothelial markers such as calretinin, CK5/6, D240, mesothelin and WT1, as well as more specific glandular markers, for example, TTF1 (lung), PAX8 (gynaecological tract, kidney, thyroid). These immunohistochemical markers have facilitated the distinction between adenocarcinoma and mesothelioma and this has resulted in a dramatic reduction in the use of EM to confirm tumour phenotype, whereas previously EM was a routine part of the diagnosis, it is now almost never performed. Additional tests to distinguish between benign and malignant mesothelial cells, including BAP1 immunohistochemistry and FISH for p16 (CDKN2A), have become available more recently; these latter tests were not in common use during the time period of the current study (Hwang et al, 2016).

As the epithelioid subtype of MM is the most common, the findings of the present study have important implications for the majority of MM cases. It is important to note that some cases of epithelioid MM cannot be diagnosed by cytology and need tissue biopsy for diagnosis, but also that tissue biopsy itself may not always accurately define MM subtype (Bueno et al, 2004),
Table 4. Variables associated with overall survival in epithelioid mesothelioma $(n=1212)$ from multivariable Cox proportional hazards model

\begin{tabular}{|l|c|c|c|c|}
\hline Variable & HR & $\begin{array}{c}\text { Lower } \\
\mathbf{9 5 \%} \mathbf{~ C l}\end{array}$ & $\begin{array}{c}\text { Upper } \\
\mathbf{9 5 \%} \mathbf{~ C l}\end{array}$ & $\mathbf{P}$ \\
\hline Age at diagnosis (per year) & 1.02 & 1.01 & 1.02 & $<0.001$ \\
\hline Peritoneal MM & 2.25 & 1.74 & 2.91 & $<0.001$ \\
\hline Male sex & 1.42 & 1.16 & 1.75 & 0.001 \\
\hline $\begin{array}{l}\text { Time from first exposure to } \\
\text { diagnosis (per year) }\end{array}$ & 0.99 & 0.99 & 1.00 & 0.03 \\
\hline Cytology diagnosed MM & 1.01 & 0.88 & 1.15 & 0.92 \\
\hline \multicolumn{4}{|l|}{ Abbreviations: $\mathrm{HR}=$ hazard ratio; $\mathrm{Cl}=$ confidence interval; MM = malignant mesothelioma. } \\
\hline
\end{tabular}

therefore, both groups in this study may contain some biphasic cases.

Cancer is a notifiable disease in WA, confirming that selection bias is unlikely as all cancer cases from across the state are notified. All diagnoses of MM were confirmed by an expert pathologist, with further case review sought to differentiate into MM subtype if required. The cohort of cytology-only diagnosed MM were older (median age 67.6 vs 70.2 years), which likely reflects different clinical practice for an older population, favouring a less invasive approach. Cox regression demonstrated that age, male sex and peritoneal $\mathrm{MM}$ had a statistically significant association with survival, but there was no significant effect of different diagnostic approach. The cases were predominantly male and occupationally exposed to asbestos, as is characteristic of this disease worldwide. Although nearly a quarter of cases were ex-Wittenoom workers or residents (with heavy exposure to exclusively crocidolite), there is no evidence to suggest that exposure to different asbestos fibre types leads to different clinical outcomes (Franklin et al, 2016). Therefore, the data are generalisable to other asbestos-exposed populations around the world.

When stratified by decade of diagnosis, the median survival increased over time with a trend towards significance (1970-1980 7.6 months, IQR 2.9-18.9 vs 2000-2012 11.8 months, IQR 6.0$20.3 ; \quad P=0.083$; Figure 2). Combined systemic pemetrexed/ cisplatin is the only therapy proven to improve overall survival in $\mathrm{MM}$ and this has been available in our institution since early 2006 (Vogelzang et al, 2003). Therefore, the trend towards increasing median survival demonstrated is likely to represent a lead-time bias with improving diagnostic expertise, approaches and increased awareness of MM by clinicians, as previously described (Musk et al, 2011). There are relatively few studies examining sensitivity of mesothelioma diagnosis by effusion cytology. Historically, sensitivities of around $30 \%$ are often quoted (Renshaw et al, 1997a), however, experienced laboratories in areas of high incidence can achieve sensitivities of over $70 \%$ (Segal et al, 2013), and other more recent studies document sensitivities of 
50-60\% (Rakha et al, 2010; Pinelli et al, 2012). The recent addition of new immunohistochemical stains (BAP1) and FISH studies $(\mathrm{CDKN} 2 \mathrm{~A} / \mathrm{p} 16)$ hold great promise for increasing the sensitivity of mesothelioma diagnosis in effusion samples in all laboratories (Walts et al, 2016).

There are a number of potential limitations of this study that should be considered when interpreting the data. It is possible that there is underrepresentation of all true MM cases in the state due to a missed diagnosis or wrong classification as a secondary pleural cancer; however, this is unlikely to proportionally represent many cases. As the cytology group was older at the time of diagnosis it is possible that they were more unwell and unable to undergo more invasive diagnostic techniques. It is also likely that some cytologically diagnosed epithelioid MM cases were in fact biphasic, however, this is equally true for histological biopsy specimens (Bueno et al, 2004). Because of the design of this study, there is no ability to assess the accuracy, sensitivity or specificity of either means of diagnostic approach; there is no record on the WAMR of if a case had the subtype changed after expert review or confirmation at post-mortem.

This study does not demonstrate that cytology-only diagnosed epithelioid MM is biologically the same as histologically diagnosed epithelioid MM. It does, however, demonstrate that there is no difference in clinically meaningful outcomes for patients using these two different diagnostic techniques. Although there are no data for treatment or disease staging, these parameters are unlikely to strongly influence the outcomes over and above cell type that is widely acknowledged to be one of the most influential variables. There is currently no evidence to suggest that there would be any difference in treatment response to chemotherapy between these groups. It is recognised that in an era of personalised medicine with genetic or immunological testing of tumour samples, there are occasions where a larger sample of (biopsy) material may be desirable. Similarly, if a patient is being considered for a study examining surgical approaches, there is also a recognised need for multiple biopsies, from both hemithoraces and mediastinum (Alvarez et al, 2009). When a diagnosis of mesothelioma is made from an effusion specimen, it is almost invariably a highly cellular sample; cases that are not shedding significant numbers of cells into the fluid will usually require biopsy for diagnosis. In these cellular fluid samples, cells shed from large areas of pleura are being collected, rather than the very limited sampling of thin core (image guided) or small biopsy samples obtained at pleuroscopy. The number of cells available in cell blocks prepared from fluid samples is thus generally far greater than that in biopsy material.

In this large cohort, this study demonstrates that the survival characteristics of cytology-only and histologically diagnosed epithelioid MM do not differ. In an experienced laboratory, a cytological diagnosis of epithelioid MM is clinically acceptable and is not associated with a clinically important different outcome when compared with histologically diagnosed cases. Therefore, a diagnosis of MM by effusion cytology may be sufficient for clinical management without the need for further invasive testing.

\section{CONFLICT OF INTEREST}

The authors declare no conflict of interest.

\section{REFERENCES}

Alvarez JM, Hasani A, Segal A, Sterret G, Millward M, Nowak A, Musk W, Bydder S (2009) Bilateral thoracoscopy, mediastinoscopy and laparoscopy, in addition to CT, MRI and PET imaging, are essential to correctly stage and treat patients with mesothelioma prior to trimodality therapy. ANZ J Surg 79(10): 734-738.
Billé A, Krug LM, Woo KM, Rusch VW, Zauderer MG (2015) Contemporary analysis of prognostic factors in patients with unresectable malignant pleural mesothelioma. J Thorac Oncol 11(2): 249-255.

Brims FJ (2009) Asbestos-a legacy and a persistent problem. J R Nav Med Serv 95(1): 4-11.

Brims FJ, Maskell NA (2013) Prognostic factors for malignant pleural mesothelioma. Curr Respir Care Rep 2(2): 100-108.

Brims FJ, Meniawy TM, Duffus I, de Fonseka D, Segal A, Creaney J, Maskell N, Lake RA, de Klerk N, Nowak AK (2016) A novel clinical prediction model for prognosis in malignant pleural mesothelioma using decision tree analysis. J Thorac Oncol 11(4): 573-582.

British Thoracic Society Standards of Care C (2007) BTS statement on malignant mesothelioma in the UK, 2007. Thorax 62(Suppl 2): ii1-ii19.

Bueno R, Reblando J, Glickman J, Jaklitsch MT, Lukanich JM, Sugarbaker DJ (2004) Pleural biopsy: a reliable method for determining the diagnosis but not subtype in mesothelioma. Ann Thorac Surg 78(5): 1774-1776.

Churg A, Colby TV, Cagle P, Corson J, Gibbs AR, Gilks B, Grimes M, Hammar S, Roggli V, Travis WD (2000) The separation of benign and malignant mesothelial proliferations. Am J Surg Pathol 24(9): 1183-1200.

Franklin P, Alfonso H, Reid A, Olsen N, Shilkin KB, Brims F, de Klerk N, Musk AW (2016) Asbestos exposure and histological subtype of malignant mesothelioma. Occup Environ Med 73(11): 749-752.

Hjerpe A, Ascoli V, Bedrossian CW, Boon ME, Creaney J, Davidson B, Dejmek A, Dobra K, Fassina A, Field A, Firat P, Kamei T, Kobayashi T, Michael CW, Onder S, Segal A, Vielh P (2015) Guidelines for the cytopathologic diagnosis of epithelioid and mixed-type malignant mesothelioma: a secondary publication. Cytopathology 26(3): 142-156.

Husain AN, Colby T, Ordonez N, Krausz T, Attanoos R, Beasley MB, Borczuk AC, Butnor K, Cagle PT, Chirieac LR, Churg A, Dacic S, Fraire A, Galateau-Salle F, Gibbs A, Gown A, Hammar S, Litzky L, Marchevsky AM, Nicholson AG, Roggli V, Travis WD, Wick M, International Mesothelioma Interest G (2013) Guidelines for pathologic diagnosis of malignant mesothelioma: 2012 update of the consensus statement from the International Mesothelioma Interest Group. Arch Pathol Lab Med 137(5): 647-667.

Hwang HC, Sheffield BS, Rodriguez S, Thompson K, Tse CH, Gown AM, Churg A (2016) Utility of BAP1 immunohistochemistry and p16 (CDKN2A) FISH in the diagnosis of malignant mesothelioma in effusion cytology specimens. Am J Surg Pathol 40(1): 120-126.

Kazan-Allen L (2005) Asbestos and mesothelioma: worldwide trends. Lung Cancer 49(Suppl 1): S3-S8.

Musk AW, de Klerk N, Eccles JL, Hobbs MS, Armstrong BK, Layman L, McNulty JC (1992) Wittenoom, Western Australia: a modern industrial disaster. Am J Ind Med 21(5): 735-747.

Musk AW, Olsen N, Alfonso H, Reid A, Mina R, Franklin P, Sleith J, Hammond N, Threlfall T, Shilkin KB, de Klerk N (2011) Predicting survival in malignant mesothelioma. Eur Respir J 38(6): 1420-1424.

Olsen NJ, Franklin PJ, Reid A, de Klerk NH, Threlfall TJ, Shilkin K, Musk B (2011) Increasing incidence of malignant mesothelioma after exposure to asbestos during home maintenance and renovation. Med J Aust 195(5): 271-274.

Pinelli V, Laroumagne S, Sakr L, Marchetti GP, Tassi GF, Astoul P (2012) Pleural fluid cytological yield and visceral pleural invasion in patients with epithelioid malignant pleural mesothelioma. J Thorac Oncol 7(3): 595-598.

Rakha EA, Patil S, Abdulla K, Abdulkader M, Chaudry Z, Soomro IN (2010) The sensitivity of cytologic evaluation of pleural fluid in the diagnosis of malignant mesothelioma. Diagn Cytopathol 38(12): 874-879.

Reid A, de Klerk NH, Magnani C, Ferrante D, Berry G, Musk AW, Merler E (2014) Mesothelioma risk after 40 years since first exposure to asbestos: a pooled analysis. Thorax 69(9): 843-850.

Renshaw AA, Dean BR, Antman KH, Sugarbaker DJ, Cibas ES (1997a) The role of cytologic evaluation of pleural fluid in the diagnosis of malignant mesothelioma. Chest 111(1): 106-109.

Renshaw AA, Nappi D, Swanson S, Sugarbaker DJ (1997b) Effusion cytology after extrapleural pneumonectomy for treatment of malignant mesothelioma. Am J Clin Pathol 107(2): 206-210.

Segal A, Sterrett GF, Frost FA, Shilkin KB, Olsen NJ, Musk AW, Nowak AK, Robinson BW, Creaney J (2013) A diagnosis of malignant pleural mesothelioma can be made by effusion cytology: results of a 20 year audit. Pathology 45(1): 44-48.

Stahel RA, Weder W, Felip E, Group EGW (2008) Malignant pleural mesothelioma: ESMO clinical recommendations for diagnosis, treatment and follow-up. Ann Oncol 19(Suppl 2): ii43-ii44. 
Vogelzang NJ, Rusthoven JJ, Symanowski J, Denham C, Kaukel E, Ruffie P, Gatzemeier U, Boyer M, Emri S, Manegold C, Niyikiza C, Paoletti P (2003) Phase III study of pemetrexed in combination with cisplatin versus cisplatin alone in patients with malignant pleural mesothelioma. J Clin Oncol 21(14): 2636-2644.

Walts AE, Hiroshima K, McGregor SM, Wu D, Husain AN, Marchevsky AM (2016) BAP1 immunostain and CDKN2A (p16) FISH analysis: clinical applicability for the diagnosis of malignant mesothelioma in effusions. Diagn Cytopathol 44(7): 599-606.
WHO (2010) World health organisation mortality database http://www. who.int/healthinfo/statistics/mortality_rawdata/en/index.html (accessed 9 January 2014).

This work is published under the standard license to publish agreement. After 12 months the work will become freely available and the license terms will switch to a Creative Commons AttributionNonCommercial-Share Alike 4.0 Unported License. 